\title{
Redox- and glucose-responsive hydrogels from poly(vinyl alcohol) and 4- mercaptophenylboronic acid
}

Article

Nurpeissova, Z. A., Alimkhanova, S. G., Mangazbayeva, R. A., Shaikhutdinov, Y. M., Mun, G. A. and Khutoryanskiy, V. V. (2015) Redox- and glucose-responsive hydrogels from poly(vinyl alcohol) and 4-mercaptophenylboronic acid. European Polymer Journal, 69. pp. 132-139. ISSN 0014-3057 doi: https://doi.org/10.1016/j.eurpolymj.2015.06.003 Available at https://centaur.reading.ac.uk/40454/

It is advisable to refer to the publisher's version if you intend to cite from the work. See Guidance on citing.

Published version at: http://www.sciencedirect.com/science/article/pii/S0014305715003079

To link to this article DOI: http://dx.doi.org/10.1016/j.eurpolymj.2015.06.003

Publisher: Elsevier

All outputs in CentAUR are protected by Intellectual Property Rights law, including copyright law. Copyright and IPR is retained by the creators or other copyright holders. Terms and conditions for use of this material are defined in the End User Agreement. 


\section{CentAUR}

Central Archive at the University of Reading

Reading's research outputs online 


\title{
Redox- and glucose-responsive hydrogels from poly(vinyl alcohol) and 4-mercaptophenylboronic acid
}

\author{
Zhansaya A. Nurpeissova, ${ }^{\text {a,b }}$ Sholpan G. Alimkhanova, ${ }^{b}$ Rauash A. Mangazbayeva, ${ }^{\text {b }}$ Yerengaip M. \\ Shaikhutdinov, ${ }^{\mathrm{b}}$ Grigoriy A. Mun ${ }^{\mathrm{b}}$ and Vitaliy V. Khutoryanskiy ${ }^{\mathrm{a}, *}$ \\ ${ }^{a}$ Reading School of Pharmacy, University of Reading, Whiteknights, PO box 224, Reading RG6 6AD, United Kingdom \\ ${ }^{b}$ al-Farabi Kazakh National University, Almaty, Kazakhstan.
}

\section{A R T ICLE INFO}

\section{Article history:}

Received

Received in revised form

Accepted

Accepted online

\section{Keywords:}

poly(vinyl alcohol)

4-mercaptophenylboronic acid

glucose-responsive hydrogel

redox-responsive hydrogel

disulfide linkage

\begin{abstract}
A B S T R A C T
Novel redox- and glucose-responsive hydrogels have been synthesized by simple mixing of poly(vinyl alcohol) (PVA) and 4-mercaptophenylboronic acid (MPBA) in aqueous solutions $(\mathrm{pH}>9)$ in an oxidative aqueous media. These hydrogels are produced through the formation of disulfide linkages between MPBA molecules in an oxidative environment (oxygen dissolved in solution or hydrogen peroxide added to the reaction mixture) and complexation via dynamic covalent bonds between PVA and MPBA dimer. These hydrogels show degradation in solutions of L-glutathione and D-glucose.
\end{abstract}

(C) 2015 Elsevier Ltd. All rights reserved.

\section{Introduction}

Hydrogels are soft, wet and elastic materials that are formed from physically or chemically cross-linked networks of hydrophilic polymers upon their swelling in water. Hydrogels have been widely studied as materials for biomedical and pharmaceutical applications. They have already found several commercial applications as contact lenses, wound dressings, drug delivery systems, hygiene products and scaffolds for tissue engineering [1-6].

Chemically cross-linked hydrogels (or chemical hydrogels) are usually synthesized either by threedimensional polymerization of hydrophilic monomers (e.g. 2hydroxy-ethylmethacrylate and 2-hydroxyethylacrylate) [7] or through cross-linking of ready-made water-soluble polymers using ionizing radiation [8,9], thermal treatment [10], or reactive low or high molecular weight cross-linkers (e.g.

*Corresponding author.

Email address: v.khutoryanskiy@ reading.ac.uk (VV.Khutoryanskiy) glutaraldehyde) [11]. Chemical hydrogels are usually irreversible, i.e. they cannot be re-dissolved because their cross-linking is achieved via stable covalent bonds [1-6].

Physically cross-linked hydrogels (physical hydrogels) are formed through non-covalent interactions either in polymers or in low molecular weight organogelators. These may include hydrogen bonding, electrostatic and hydrophobic effects, crystallization, etc. Examples of physical hydrogels include cryogels formed by poly(vinyl alcohol) (PVA) using freeze-thawing technique to induce formation of crystallites that act as physical cross-links [12]; thermally-reversible gels formed by some block-copolymers [13], hydrogels formed by reaction of alginate with calcium ions [14], and gels formed by self-assembly of certain peptide-based hydrogelators [15]. Physical hydrogels are typically reversible, i.e. they could be re-dissolved in response to changes in environmental conditions (e.g. temperature, $\mathrm{pH}$ of solution, etc).

Boronic acid-containing hydrogels have been recently recognized as an important class of intelligent materials due to their unique properties such as glucose-sensitivity, reversibility and self-healing ability [16]. These hydrogels 
could be formed through interaction between boronic acid containing molecules and poly(vinyl alcohol) in aqueous solutions. This interaction is covalent in nature but the bonds formed are not very stable and are reversible (dynamic covalent bonds) upon changes in environmental temperature, $\mathrm{pH}$ and presence of competitive molecules (e.g. glucose). These materials represent an intermediate class between chemical and physical hydrogels because of their covalent nature of cross-linking but reversibility in their properties. Hydrogels formed in aqueous mixtures of PVA and borate ions were considered as materials promising for pharmaceutical applications [17].

Recently, Yang and co-workers [18] reported the formation of glucose-responsive hydrogels based on dynamic covalent chemistry and inclusion complexation. They have synthesized bifunctional phenylboronic acid (PBA)terminated cross-linker based on polyethyleneglycol (PBAPEG-PBA) and formed gels by mixing this material with poly(ethylene oxide)-b-poly(vinyl alcohol) (PEG-b-PVA) and $\alpha$-cyclodextrin in aqueous solutions. The hydrogel was formed through the interactions of PVA blocks with PBAPEG-PBA and inclusion complexation between PEO blocks and $\alpha$-cyclodextrin.

Chemical reactions involving thiol functional groups ($\mathrm{SH})$ have recently received a lot of interest from material and polymer scientists. Thiol groups have excellent reactivity that allows their use in a variety of reactions leading to new materials, polymers or bioconjugates $[19,20]$. One of the unique features of thiol groups is their ability to undergo oxidation under very mild conditions to form disulfide bonds (-S-S-). This oxidation could be mediated by atmospheric oxygen or by the presence of oxidizing agents in solution, e.g. hydrogen peroxide $[21,22]$.

Here, we report a novel one-pot synthesis of hydrogels in aqueous mixtures of PVA and 4-mercaptophenylboronic acid (MPBA) in the presence of an oxidative environment (atmospheric oxygen or hydrogen peroxide). The oxidative environment facilitated the formation of MPBA dimers via disulfide bringing that act as cross-linker for PVA via dynamic covalent chemistry. The disulfide bridges (-S-S-) in these hydrogels could be easily cleaved in the presence of glutathione, which makes these materials sensitive to redox environment. The bonds formed between $-\mathrm{B}(\mathrm{OH})_{2}$ groups of MPBA and 1,2-diols of PVA could also be cleaved in the presence of D-glucose, making the hydrogels glucoseresponsive.

\section{Experimental section}

\subsection{Materials}

Two batches of poly(vinyl alcohol) (PVA) were used in this study: 88-97 kDa PVA, 87-89\% hydrolyzed (Alpha Aesar) and 205 kDa PVA 86.7-88.7 \% hydrolyzed (FlukaChemika). 4-mercaptophenylboronic acid (MPBA, purity $\geq 90 \%$ ), L-glutathione reduced (purity $\geq 98 \%$ ) and hydrogen peroxide (30\%) were purchased from Sigma Aldrich, Inc. (UK); D-glucose was received from $\mathrm{BDH}$ Analar; sodium hydroxide (analytical grade 97+\%) was purchased from Fisher Scientific. All reagents were used as received.

\subsection{Preparation of solutions}

PVA solutions of three concentrations (4, 6, and $10 \mathrm{w} / \mathrm{v}$ $\%)$ were prepared by dissolving dry polymer in deionized water at $80^{\circ} \mathrm{C}$ with constant stirring for 5 hours. Then these solutions were stirred at room temperature overnight. MPBA solutions were freshly prepared prior to each experiment in 0.1-1 $\mathrm{M}$ aqueous solutions of $\mathrm{NaOH}$.

\subsection{Rheological measurements}

The rheological properties of PVA-MPBA gels were studied using an AR 2000 EX Rheometer (TA Instruments, UK) with $40.0 \mathrm{~mm}$ diameter parallel plate geometry and 1 $\mathrm{mm}$ gap distance. Dynamic time sweep experiments were carried out with a frequency and strain of $1 \mathrm{rad} / \mathrm{s}$ and $5 \%$, respectively. To perform each experiment $0.5 \mathrm{~mL}$ of $6 \mathrm{w} / \mathrm{v} \%$ PVA solution was mixed with $0.5 \mathrm{~mL}$ of $1 \mathrm{w} / \mathrm{v} \%$ MPBA solutions in different concentrations of $\mathrm{NaOH}(0.1 \mathrm{M}, 0.5 \mathrm{M}$, $1 \mathrm{M})$ and $0.67 \mathrm{~mL}$ of mixture was immediately loaded into the rheometer. In some experiments $0.3 \mathrm{~mL}$ of $1 \% \mathrm{H}_{2} \mathrm{O}_{2}$ was also added to facilitate oxidation of $-\mathrm{SH}$ groups in MPBA. Frequency sweep experiments were performed with a frequency and strain of $0.1-100 \mathrm{rad} / \mathrm{s}$ and $5 \%$, respectively. For this experiment $0.5 \mathrm{~mL}$ of different concentrations of MPBA solutions $(0.5,1,2,3,4$, and $5 \mathrm{w} / \mathrm{v} \%)$ in $1 \mathrm{M} \mathrm{NaOH}$ were used. They were mixed with $6 \mathrm{w} / \mathrm{v} \%$ of PVA solutions and shaken during 28 hours. All rheological experiments were conducted at $25^{\circ} \mathrm{C}$.

\subsection{Scanning electron microscopy (SEM)}

The morphology of the freeze dried samples of PVA solutions and the hydrogels were examined with FEI Quanta FEG 600 scanning electron microscope (Hillsboro, UK) at acceleration voltage of $10 \mathrm{kV}$. The hydrogel samples were prepared by mixing $1 \mathrm{~mL}$ of 4,6 , and $10 \mathrm{w} / \mathrm{v} \%$ PVA solutions with $1 \mathrm{~mL}$ of $1 \mathrm{w} / \mathrm{v} \%$ MPBA solution (in $0.1 \mathrm{M}$ $\mathrm{NaOH}$ ). PVA solutions mixed with $1 \mathrm{~mL}$ of $0.1 \mathrm{M} \mathrm{NaOH}$ were used as non-cross-linked control samples. The hydrogels and control samples were shaken for 5 hours at room temperature and then left undisturbed overnight. Afterwards, all samples were freeze dried (Thermo LL3000) under identical conditions. Each freeze-dried sample was sputter coated with gold prior to SEM experiments.

\subsection{Swelling measurements}

Swelling measurements with PVA-MPBA hydrogels were carried out in deionized water and 1,5 , and $7 \mathrm{mM}$ solutions of 
L-glutathione, and in 10, 100 and $250 \mathrm{mM}$ solutions of Dglucose. For these particular experiments the hydrogels were prepared by mixing $1 \mathrm{~mL}$ of $6 \mathrm{w} / \mathrm{v} \%$ PVA solution with 1 $\mathrm{mL}$ of $2 \mathrm{w} / \mathrm{v} \%$ MPBA solution (in $0.25 \mathrm{M} \mathrm{NaOH}$ ) and shaking the mixtures for 5 hours. Then the hydrogels were kept undisturbed for 5 days at room temperature prior to swelling experiments. Longer storage of these hydrogels often results in formation of samples with intensive colour, which is likely related to oxidation processes. These samples were immersed in solutions and weighed out every 24 hours during 6 days. Their swelling was quantitatively assessed through the swelling ratio (SR):

$$
\mathrm{SR}=\mathrm{m}_{2} / \mathrm{m}_{1}
$$

where $m_{1}$ is the weight of the hydrogel after the synthesis and $\mathrm{m}_{2}$ is the weight of the sample after its immersion into deionized water or L-glutathione. In a separate set of experiments the hydrogel samples were immersed in a swelling medium (deionized water or $5 \mathrm{mM}$ solutions of Lglutathione) in Petri dishes with a gentle stirring and images were taken every day for 6 days.

\section{Results and discussion}

\subsection{Formation of hydrogels}

MPBA is a relatively hydrophobic compound that is soluble in water only at $\mathrm{pH}>9$, so all experiments used $0.1-1$ $\mathrm{M} \mathrm{NaOH}$ solutions as a medium.

Mixing of aqueous solutions of PVA and MPBA at different concentrations resulted in the formation of hydrogels, which was initially detected visually through the pronounced thickening of the mixture with a subsequent formation of a non-flowing gel. The experiments were performed using rheological measurements and provided a quantitative data on the gelation process. Fig. 1 shows the exemplar rheological profile of PVA-MPBA mixture as a function of time.

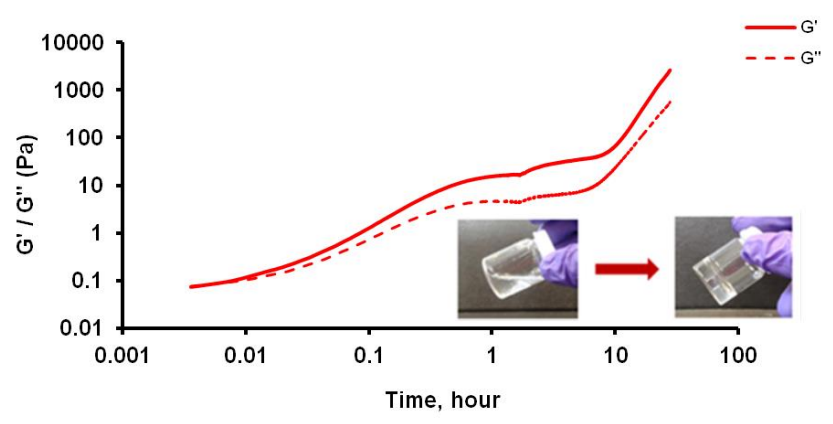

Fig. 1. Exemplar rheological profile of the reaction mixture containing $6 \mathrm{w} / \mathrm{v} \%$ PVA (88-97kDa) and $2 \mathrm{w} / \mathrm{v} \%$ MPBA (prepared in $0.25 \mathrm{~mol} / \mathrm{L} \mathrm{NaOH})$. Insert: Photograph of the gelation in this reaction mixture (time $=28$ hours).
The initial values of the loss modulus G', and the storage modulus $G^{\prime}$ are relatively low and are roughly equal, which indicates that in the beginning of the cross-linking the reaction mixture exhibits a liquid flow behaviour. Both $G$ ', and $G^{\prime}$ show a tendency to increase with time and $G^{\prime}>G^{\prime}$ ', which indicates the formation of a gel [23]. These experiments were performed with PVA and MPBA solutions with access to atmospheric oxygen, which is believed to play a role in inducing the dimerization of MPBA via disufide bridge formation [21, 22, 24, 25].

Similar or even more intensive gelation is observed when small portions of hydrogen peroxide were added to the mixtures of PVA and MBPA (Fig. 1S, ESI). Hydrogen peroxide facilitates the dimerization of MPBA through the oxidation of $-\mathrm{SH}$ groups and formation of disulfide bridges. A series of control experiments were also performed to prove that the gelation in PVA-MBPA mixtures is related to the dimerization of MPBA via oxidation resulting in subsequent cross-linking of PVA.

Further rheological experiments were performed to establish the effect of $\mathrm{NaOH}$ concentration on gelation. Fig. 2 presents the rheological profiles recorded for gelation in PVA-MPBA mixtures in the presence of $\mathrm{NaOH}$ of different concentrations. The gelation in PVA-MPBA mixtures in 0.1 $\mathrm{M} \mathrm{NaOH}$ solutions is observed almost immediately ( $\mathrm{G}^{\prime}>\mathrm{G}^{\prime}$ '), and both G' and G', grow with time leading to gradual mechanical strengthening of the hydrogel. At higher concentrations of $\mathrm{NaOH}(0.5$ and $1 \mathrm{M} \mathrm{NaOH})$ no gelation is detected initially after reagents mixing; then a rapid growth of both G' and G', is observed after $\sim 1$ hour and $\sim 2 \mathrm{hrs}$ in $0.5 \mathrm{M}$ and $1 \mathrm{M} \mathrm{NaOH}$, respectively. This rapid growth in $\mathrm{G}^{\prime}$ and $\mathrm{G}^{\prime}$ ', also indicates the formation of hydrogels. The growth of $\mathrm{G}^{\prime}$ and G' values with time also shows the presence of some features of unclear nature. It could be either related to the artefacts produced by a rheometer in time sweep measurements or the cross-linking reactions proceeds through several stages (e.g. cross-linking caused by PVA reaction with MPBA dimer; oxidation of MPBA monomer, etc).

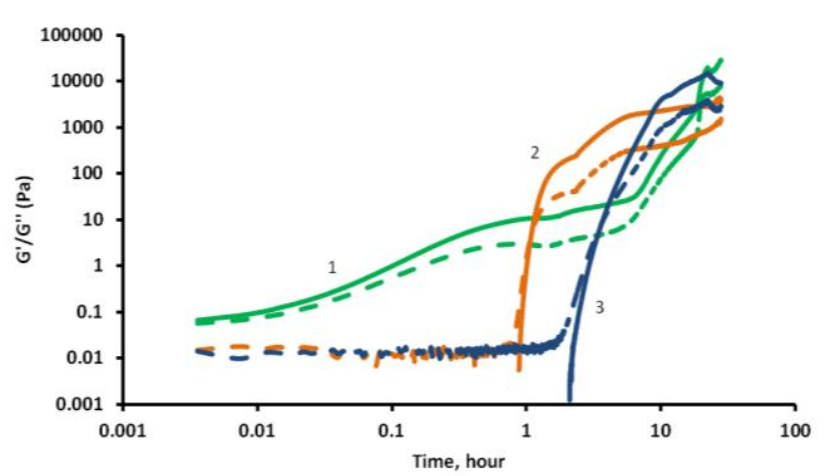

Fig. 2. Rheological profiles with G' (solid line) and G' (dashed line) for the reaction mixtures containing $6 \mathrm{w} / \mathrm{v} \%$ PVA $(88-97 \mathrm{kDa})$ and 1 w/v \% MPBA prepared in $0.1 \mathrm{M}$ (1, green), $0.5 \mathrm{M}$ (2, orange) and 1 $\mathrm{M} \mathrm{NaOH}$ (3, blue). 
The concentration of $\mathrm{NaOH}$ could potentially have an effect on oxidation and formation of S-S bonds as well as on the formation of dynamic covalent bonds between 1,2-diols and$\mathrm{B}(\mathrm{OH})_{2}$ groups. The elucidation of the kinetics of crosslinking in these systems will require a separate detailed study. In a control experiment the solution of $6 \mathrm{w} / \mathrm{v} \%$ PVA was mixed with $0.1 \mathrm{M} \mathrm{NaOH}$ and no gelation was observed within 28 hours; this solution remained in a liquid state with only a very modest increase in both $G^{\prime}$ ' and $G^{\prime}$ 'values with time (Fig. 2S, ESI). This modest increase in G' ' and G' may be caused by hydrogen bonding between undisturbed macromolecules of PVA in solutions.

The rheological studies continued to look at the effect of MPBA concentration on the gelation in PVA-MPBA mixtures. These experiments were performed at a wide range of oscillation frequencies. The exemplar frequency sweep is presented in Fig. 3s (ESI). Fig. 3 shows the changes in G', and $G^{\prime}$ values as a function of MPBA concentration in the mixture at $\omega=10 \mathrm{rad} / \mathrm{s}$ (note that $\omega$ here is different to $\omega=1$ $\mathrm{rad} / \mathrm{s}$ in time sweep experiments shown in Figures 1 and 2). No gelation was observed when MPBA concentration was below $0.5 \mathrm{w} / \mathrm{v} \%$. An increase in MPBA concentration in PVA/MPBA mixture from 0.5 to $2 \mathrm{w} / \mathrm{v} \%$ results in the growth of $G^{\prime}$ ' and $G^{\prime}$ until they reach saturation at $\mathrm{C}(\mathrm{MBPA})>2 \mathrm{w} / \mathrm{v} \%$. The further increase in MPBA concentration does not change the mechanical properties of the hydrogels. This saturation is achieved when PVA / MPBA molar ratio is around $2: 10 \mathrm{~mol} / \mathrm{mol}$.

The structural features of the hydrogels formed as a result of the reaction between PVA and MPBA were studied using scanning electron microscopy (Fig. 4). For this purpose PVA solutions as well as PVA-MPBA hydrogels were freeze-dried under identical conditions and investigated using SEM.

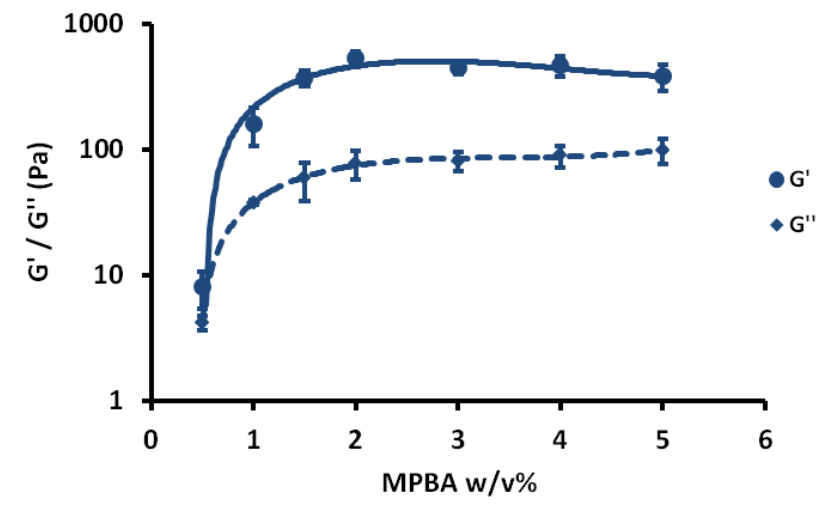

Fig. 3. Effect of MPBA concentration in the reaction mixture on the rheological properties of PVA-MPBA gels recorded at $\omega=10 \mathrm{rad} / \mathrm{s}$ and $5 \%$ strain. PVA $(88-97 \mathrm{kDa})$ concentration is $6 \mathrm{w} / \mathrm{v} \%$. MPBA solutions were prepared in $1 \mathrm{M} \mathrm{NaOH}$. PVA-MPBA mixtures were prepared and shaken for 28 hours prior to this experiment. Each experiment was performed in triplicate and the results are presented as a mean value \pm standard deviation
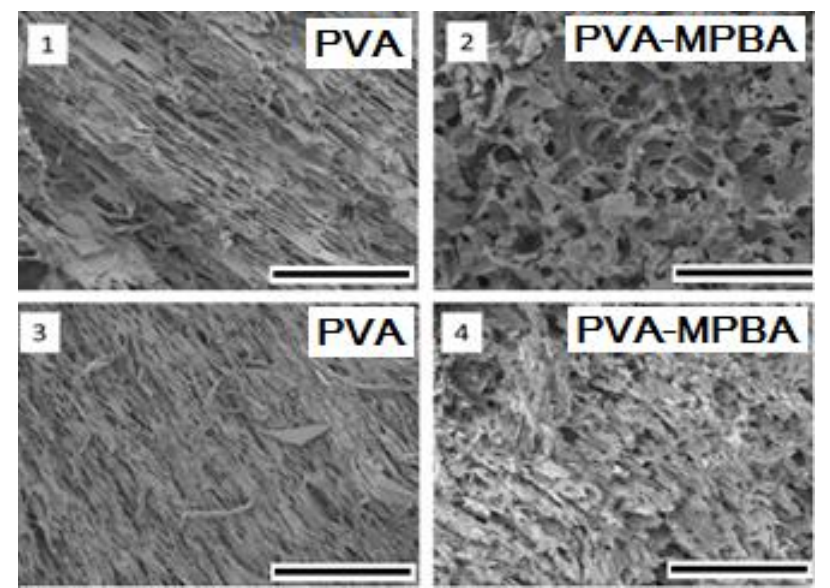

Fig. 4. SEM images of freeze-dried $6 \mathrm{w} / \mathrm{v} \%$ PVA (1) and $10 \mathrm{w} / \mathrm{v} \%$ PVA (3) and the hydrogels formed in the mixtures of $6 \mathrm{w} / \mathrm{v} \% \mathrm{PVA}-$ $1 \mathrm{w} / \mathrm{v} \%$ MPBA (2) and $10 \mathrm{w} / \mathrm{v} \%$ PVA - $1 \mathrm{w} / \mathrm{v} \%$ MPBA (4). MPBA solutions were prepared in $0.1 \mathrm{M} \mathrm{NaOH}$. PVA (88-97kDa) was used in this experiment. Size bar is $300 \mu \mathrm{m}$.

It can be clearly seen that the PVA cross-linked with MBPA has a porous structure distinctly different from the morphology of freeze-dried PVA solutions.

All the above results allow us to propose the following scheme for the formation of hydrogels in PVA/MPBA mixtures (Fig. 5). Commercial MPBA is always contaminated with its dimer formed as a result of oxidation. The presence of MPBA dimer initiates the gelation process of PVA and the gels become mechanically stronger with time as a result of further cross-linking due to the oxidation and dimerization of MPBA in the presence of oxidising species (either atmospheric oxygen or $\mathrm{H}_{2} \mathrm{O}_{2}$ ). The MPBA dimer acts as a bifunctional cross-linker that reacts with 1,2-diol groups in PVA and results in formation of dynamic covalent bonds.

\subsection{Redox- and glucose-responsive properties}

The presence of disulfide bonds in MPBA dimer-based cross-links between PVA macromolecules in these hydrogels provides an interesting opportunity for gradual degradation / dissolution of these materials in the reductive environment, typical for some biological systems. For example, glutathione is an important biological reducing agent that is present in animal cells in millimolar concentrations [26]. A degradation of hydrogels cross-linked via disulfide bonds has previously been reported in glutathione solutions by Choh et al. [27]. Glutathione has also been demonstrated to trigger disassembly of polymer nanoparticles, formed from thermoresponsive polymers containing a disulfide-linked 'solubility release catch' due to cleavage of disulfide bonds [28].

In this study, we have demonstrated that the presence of glutathione in solution at physiologically-relevant concentrations results in a gradual degradation of PVAMPBA hydrogels. 


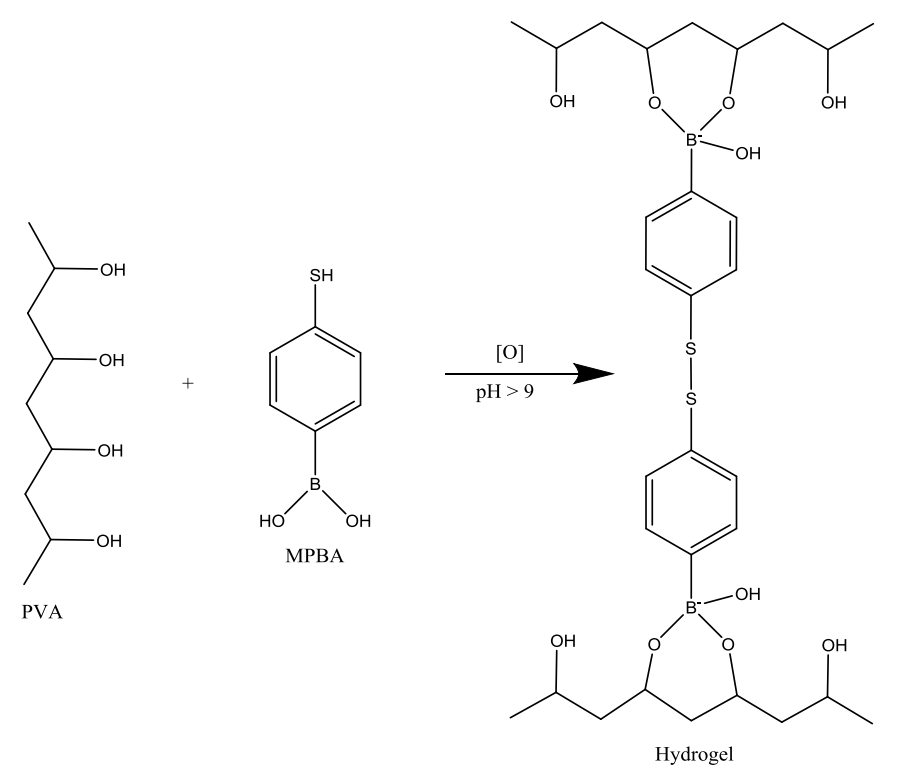

Fig. 5. Proposed scheme of PVA cross-linking with MPBA in an oxidative environment.

This degradation is distinctly different from additional swelling and partial dissolution of these samples in deionized water. The PVA-MPBA samples immersed in deionized water initially undergo additional swelling and then reduce their volume due to partial dissolution of non-cross-linked PVA macromolecules physically trapped within the chemically cross-linked network (Fig. 6). When the same hydrogels are exposed to $5 \mathrm{mM}$ solutions of L-glutathione their initial swelling is much less pronounced and the samples show a gradual size reduction and degradation into smaller pieces. Note that a disappearance of a yellow colour upon samples swelling and degradation is likely related to the release of coloured small molecular weight oxidation by-products.

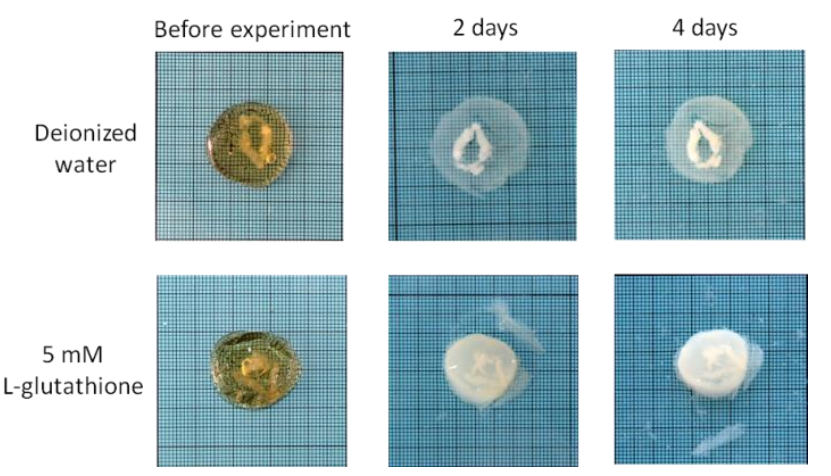

Fig. 6. Swelling and degradation of PVA-MPBA hydrogels in deionized water and $5 \mathrm{mM}$ solutions of L-glutathione. These hydrogels were prepared from $6 \mathrm{w} / \mathrm{v}$ PVA $(205 \mathrm{kDa})$ and $2 \mathrm{w} / \mathrm{v} \%$ MPBA in $0.25 \mathrm{M} \mathrm{NaOH}$
The quantitative experiments on swelling/disintegration of PVA-MPBA hydrogels performed gravimetrically also demonstrate the same trend (Fig. 7). The PVA-MPBA samples immersed in deionized water undergo additional swelling for 2 days and then subsequently show a weight reduction. The samples immersed in solutions 1,5 and $7 \mathrm{mM}$ solutions of L-glutathione show less intensive swelling and then more intensive weight loss associated with the sample gradual degradation. It should be noted that the swelling/degradation profiles of these hydrogels are highly dependent on a number of factors such as the grade of PVA used (molecular weight and degree of deacetylation) and conditions used for PVA cross-linking (e.g. cross-linking time, exposure to atmospheric oxygen or to hydrogen peroxide).

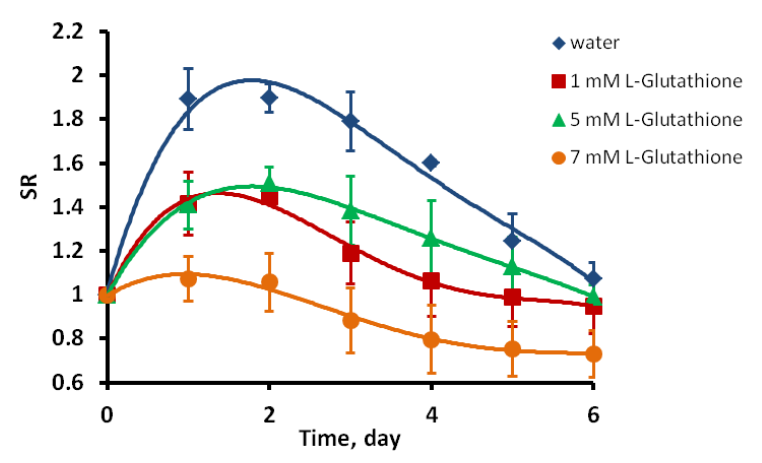

Fig. 7. Quantitative experiments on swelling/degradation of PVAMPBA hydrogels in deionized water and solutions of L-glutathione. These hydrogels were prepared from $6 \mathrm{w} / \mathrm{v}$ PVA (205 kDa) and $2 \mathrm{w} / \mathrm{v}$ $\%$ MPBA in $0.25 \mathrm{M} \mathrm{NaOH}$. Each experiment was performed in triplicate and the results are presented as the mean value \pm standard deviation. 
Boronic acid-containing PVA hydrogels are also known to show glucose-responsive properties due to the ability of small sugar molecules to compete with 1,2-diols of PVA for complexation/dynamic covalent bonds with $\mathrm{B}(\mathrm{OH})_{2}$ - groups [16]. Our experiments on the swelling of PVA-MPBA hydrogels have shown that the hydrogels undergo gradual degradation in solutions of D-glucose (Fig. 8). Some hydrogel degradation is observed in $10 \mathrm{mM}$ solution of D-glucose, which corresponds to its physiological concentration in human blood [29] The degradation of hydrogels becomes even more pronounced with increase in the concentration of D-glucose in solutions, e.g. in $250 \mathrm{mM}$ solutions.

Fig. 9 shows the proposed mechanism of hydrogel degradation in the presence of L-glutathione and D-glucose. L-glutathione causes the cleavage of disulfide bonds in MPBA dimers, acting as cross-links in the hydrogels, whereas D-glucose competes with 1,2-diols of PVA for the complexation with $\mathrm{B}(\mathrm{OH})_{2}$-groups of MPBA dimers.

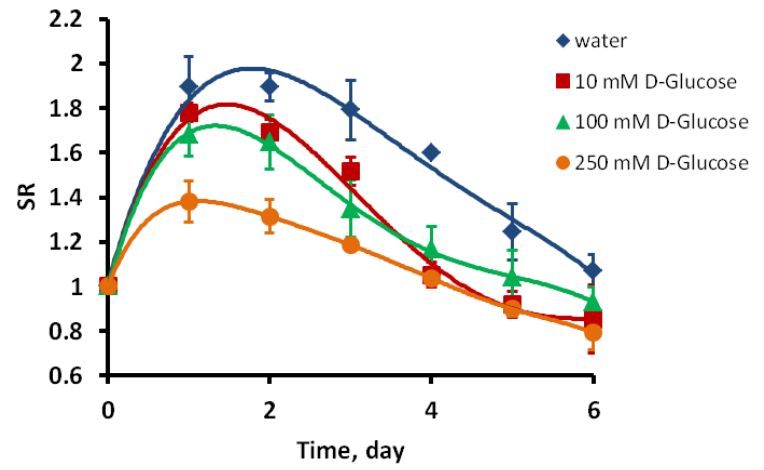

Fig. 8. Quantitative experiments on swelling/degradation of PVAMPBA hydrogels in deionised water and solutions of D-glucose. These hydrogels were prepared from $6 \mathrm{w} / \mathrm{v}$ PVA $(205 \mathrm{kDa})$ and $2 \mathrm{w} / \mathrm{v}$ $\%$ MPBA in $0.25 \mathrm{M} \mathrm{NaOH}$. Each experiment was performed in triplicate and the results are presented as the mean value \pm standard deviation

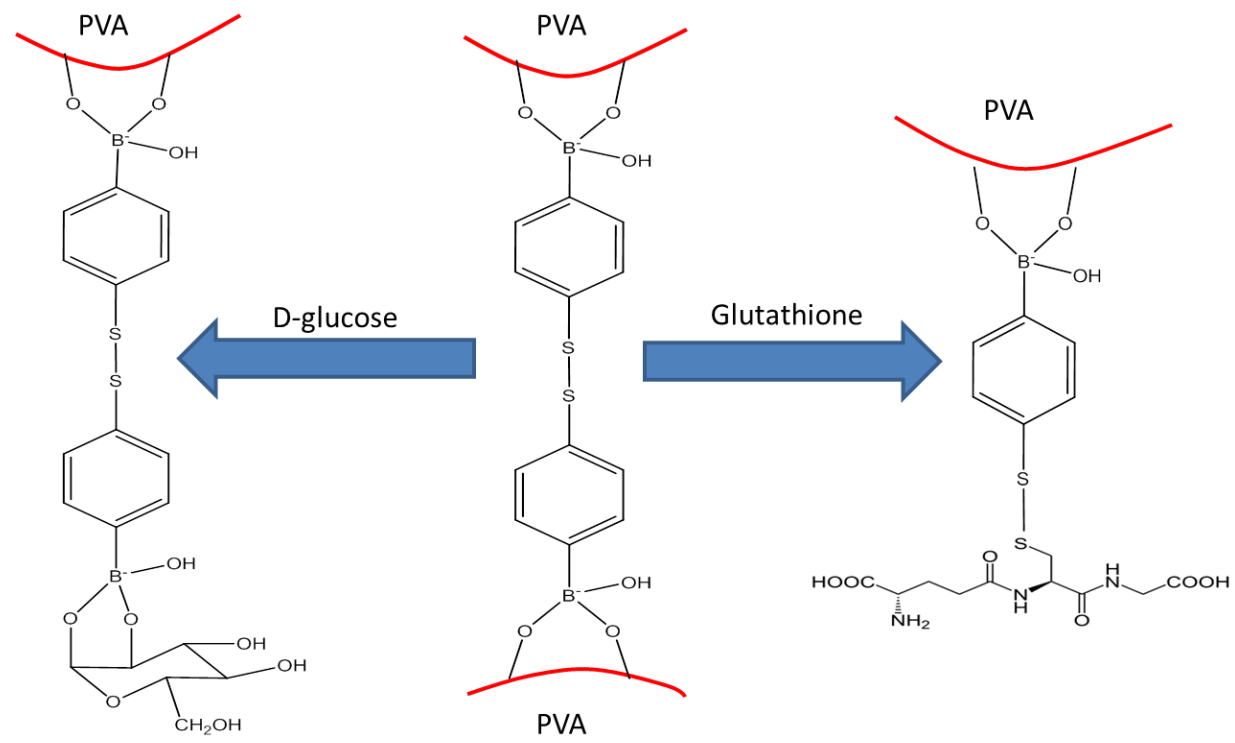

Fig. 9. Mechanisms of degradation of PVA-MPBA hydrogels in the presence of L-glutathione and D-glucose.

The complete degradation/dissolution of these hydrogels in solutions of L-glutathione and D-glucose is not observed because of the specific nature of PVA. It is well known that PVA is a semi-crystalline polymer, whose solubility in water depends on its molecular weight and degree of deacetylation [30]. Some grades of PVA will require initial heating to approximately $80-85^{\circ} \mathrm{C}$ to dissolve it in water; this is needed to disrupt crystalline domains and hydrogen bonds, acting as physical cross-links and holding PVA macromolecules together. Once aqueous solution is formed it remains relatively stable, but may thicken with time due to reformation of intermacromolecular hydrogen bonds. If this solution is frozen and then carefully thawed, the physically cross-linked hydrogels may be formed due to intermacromolecular hydrogen bonds and formation of crystallites, acting as physical cross-links (this principle is used to form so-called cryogels from PVA) [12]. It could be hypothesized that incomplete degradation of PVA-MPBA hydrogels in solutions of L-glutathione or D-glucose is also related to the formation of additional cross-linking junctions between PVA macromolecules due to hydrogen bonding / crystallites.

\section{Conclusions}

Simple mixing of aqueous solutions of poly(vinyl alcohol) with 4-mercaptophenylboronic acid at $\mathrm{pH}>9$ in an oxidative environment (either presence of hydrogen peroxide or exposure to atmospheric oxygen) results in formation of polymeric hydrogels. These hydrogels are formed due to 
oxidative dimerization of 4-mercaptophenylboronic acid via disulfide bond formation, and dynamic covalent bond formation between 1,2-diols present in poly(vinyl alcohol) and phenylboronic acid groups. These hydrogels undergo a gradual degradation in solutions of L-glutathione and Dglucose.

\section{Acknowledgements}

This work was partially supported by the grant from the Ministry of Education and Science of the Republic of Kazakhstan (3471/GF4).

\section{Appendix A. Supplementary material.}

Rheological profile for PVA-MPBA hydrogels formed in the presence of hydrogen peroxide; exemplary frequency sweep of PVA-MPBA hydrogels; rheological profile of a PVA mixed with solution of $\mathrm{NaOH}$ (control experiment).

\section{References}

[1] Y. Qiu, K. Park, Adv. Drug Deliv. Reviews, 2001, 53, 321.

[2] S. Hoffman, Adv. Drug Deliv. Reviews, 2002, 54, 3.

[3] N. A. Peppas, J. Z. Hilt, A. Khademhosseini, R. Langer, $A d v$. Materials, 2006, 18, 1345.

[4] T.R.Hoare, D.S. Kohane, Polymer, 2008, 49, 1993.

[5] W.E. Hennink, C.F. Van Nostrum, Adv. Drug Deliv. Reviews, 2012, 64, 223.

[6] E. Calo, V.V. Khutoryanskiy, Eur. Polym. J., 2015, 65, 252.

[7] O.V. Khutoryanskaya, Z.A. Mayeva, G.A. Mun, V.V. Khutoryanskiy, Biomacromolecules, 2008, 9, 3353.

[8] J.M. Rosiak, J. Controlled Release, 1994, 31, 9.

[9] Z.S. Nurkeeva, G.A. Mun, A.V. Dubolazov, V.V. Khutoryanskiy, Macromol. Biosci., 2005, 5, 424.
[10] J.P. Cook, G.W. Goodall, O.V. Khutoryanskaya, V.V. Khutoryanskiy, Macromol. Rapid Commun.,2012, 33, 332.

[11] A. Jayakrishnan, S.R. Jameela, Biomaterials, 1996, 17, 471.

[12] C.M. Hassan, N.A. Peppas, Macromolecules 2000, 33, 2472.

[13] M.A. Ward, T.K. Georgiou, Polym. Chem., 2013, 4, 1893

[14] K.Y. Lee, D.J. Mooney, Prog Polym Sci., 2012, 37, 106.

[15] D.J. Adams, P.D. Topham, Soft Matter, 2010, 6, 3707.

[16] Y. Guan, Y. Zhang, Chem. Soc. Rev., 2013, 42, 8106.

[17] R.G. Loughlin, M.M. Tunney, R.F. Donnelly, D.J. Murphy, M. Jenkins, P.A. McCarron, Eur. J. Pharm. Biopharm., 2008, 69, 1135.

[18] T. Yang, R. Ji, X.-X. Deng, F-S. Du, Z.-C. Li, Soft Matter, 2014, 10, 2671.

[19] M.H. Stenzel, ACS MacroLett., 2012, 2, 14.

[20] M. Le Neindre, R. Nicolay, Polym. Chem., 2014, 5, 4601.

[21] V.V. Khutoryanskiy, N. Tirelli, Pure Appl. Chem., 2008, 80, 1703.

[22] G.S. Irmukhametova, G.A. Mun, V.V. Khutoryanskiy, Langmuir, 2011, 27, 9551.

[23] M. Djabourov, J. Leblond, P. Papon, J. Phys. France, 1988, 49, 333.

[24] L Aravindan, K.A. Bicknell, G. Brooks, V.V. Khutoryanskiy, A.C. Williams, Macromol. Biosci., 2013, 13, 1163.

[25] K. Kafedjiiski, F. Föger, H. Hoyer, A. Bernkop-Schnürch, M. Werle, Drug Devel. Ind. Pharm., 2007, 33, 199.

[26] D. Montero, C. Tachibana, J.R. Winther, C. Appenzeller-Herzog, Redox Biology, 2013, 1, 508.

[27] S.-Y. Choh, D. Cross, C. Wang, Biomacromolecules, 2011, 12, 1126.

[28] D.J. Phillips, J.P. Patterson, R.K. O'Reilly, M.I. Gibson, Polym. Chem., 2014, 5, 126.

[29] S. Joel, K.B. Turner, S. Daunert, ACS Chem. Biol. 2014, 9, 1595.

[30] M.I. Baker, S.P. Walsh, Z. Schwartz, B.D. Boyan, J. Biomed. Mater. Res. B Appl. Biomater., 2012, 100, 1451. 